\title{
Characterization of Peripheral Anterior Synechiae Formation After Microhook Ab-interno Trabeculotomy Using a 360-Degree Gonio-Camera
}

\section{Masato Matsuo \\ Yuina Inomata \\ Nana Kozuki \\ Masaki Tanito}

Department of Ophthalmology, Shimane University Faculty of Medicine, Izumo,

Shimane, 693-850I, Japan
Correspondence: Masato Matsuo Department of Ophthalmology, Shimane University Faculty of Medicine, Enya 89-I, Izumo, Shimane, 693-850I, Japan

Tel +8I-853-20-2284

Fax +8I-853-20-2278

Email matsuohp@med.shimane-u.ac.jp
Purpose: To investigate the prevalence, locations, and characteristics of peripheral anterior synechiae (PAS) formation after microhook ab-interno trabeculotomy ( $\mu$ LOT), a minimally invasive glaucoma surgery, using a 360-degree gonio-camera, gonioscope GS-1 (NIDEK Co., Gamagori, Japan).

Subjects and Methods: A total of 105 consecutive eyes of 75 subjects with open-angle glaucoma were analyzed. The eyes had undergone $\mu \mathrm{LOT}$ or combined $\mu \mathrm{LOT}$ and cataract surgery as an initial glaucoma surgery. Postoperative PAS formation was evaluated in 16 iridocorneal angle images with the best focus covering 360 degrees in each eye.

Results: Compared to baseline, at $225 \pm 226$ days postoperatively, the intraocular pressure and number of antiglaucoma medications decreased significantly $(\mathrm{p}<0.01$, respectively). PAS developed in $86 \%$ of eyes. The mean number of iridocorneal angle images that showed PAS in all eyes was $4.1(26 \%)$ in the total circumference, $3.1(39 \%)$ within the $\mu$ LOT incision, and $1.0(13 \%)$ outside of the $\mu$ LOT incision; the rate was significantly $(\mathrm{p}<0.0001)$ higher within the incision than outside of the incision. Moreover, the higher total PAS rate and that within the incision may be associated with later postoperative days ( $p=0.01$ and 0.004 , respectively), that outside of the incision with $\mu \mathrm{LOT}$ alone rather than the combined surgery, and with shallower preoperative central anterior chamber depth $(\mathrm{p}=0.048$ and 0.04 , respectively), calculated by the mixed-effect model.

Conclusion: The current results showed the characteristics of PAS formation after $\mu$ LOT using 360-degree gonio-images, and the formation rate was significantly higher within the $\mu$ LOT incision. The PAS within and outside of the incision had different causes.

Keywords: peripheral anterior synechiae, microhook ab-interno trabeculotomy, minimally invasive glaucoma surgery, open-angle glaucoma, 360-degree gonio-camera

\section{Introduction}

Glaucoma is the leading cause of irreversible blindness worldwide, and the global number of affected individuals is expected to increase with the growing elderly population. ${ }^{1,2}$ Because elevated intraocular pressure (IOP) is a major risk factor for glaucoma development and progression, ${ }^{3}$ it is necessary for IOP to be controlled with medications, laser treatment, and surgery. Ab-externo filtration surgery such as trabeculectomy is remarkably effective and considered the gold standard; however, it may lead to significant complications, the consideration of which can sometimes 
delay glaucoma surgery until all other less invasive treatment options are attempted. Various minimally invasive glaucoma surgery (MIGS) procedures are safer and less traumatic with an ab-interno approach for patients with mild-to-moderate glaucoma or those who are refractory to standard medical therapy. ${ }^{4}$ Therefore, MIGS could decrease the number of patients who require more invasive surgeries to control the IOP. These surgeries also lowered the hurdles for surgical intervention to provide better IOP control and reduce the glaucoma medication burden. Abinterno trabeculotomy is a MIGS that increases trabecular outflow $^{4-8}$ and is increasing in popularity. The surgical mechanism is assumed to be elimination of aqueous flow resistance by cleavage of the trabecular meshwork (TM) and inner walls of Schlemm's canal at the point of outflow resistance. ${ }^{9,10}$ However, peripheral anterior synechiae (PAS) may develop after ab-externo trabeculotomy (modified 360-degree suture trabeculotomy) that obstruct the TM, decrease the outflow, and possibly weaken the IOPlowering effect. ${ }^{11,12}$ However, PAS formation after trabeculotomy has not been studied in detail.

Microhook ab-interno trabeculotomy $(\mu \mathrm{LOT})$ is a novel ab-interno trabeculotomy-related glaucoma surgery in which Tanito ab-interno Trabeculotomy Microhooks (Inami \& Co., Ltd., Tokyo, Japan) are used to incise the TM; several studies have reported the effectiveness of $\mu \mathrm{LOT}$ for decreasing both the IOP and number of antiglaucoma medications used..$^{9,10,13-15}$ However, PAS also develop after $\mu \mathrm{LOT}$, which we reported after evaluating images of the entire circumference anterior chamber angle using the Gonioscope GS-1 (NIDEK Co., Gamagori, Japan). ${ }^{16}$ Postoperative PAS formation after $\mu$ LOT also is not well studied; however, it could potentially reduce the surgical effectiveness and therefore would be clinically relevant. In addition, investigation of this would contribute to a better understanding of the surgical outcomes of trabeculotomy. Thus, the purpose of the current study was to investigate the prevalence, locations, and characteristics of PAS formation after $\mu$ LOT using the GS-1 and evaluate the effects on various clinical variables including IOP and number of antiglaucoma medications.

\section{Subjects and Methods}

The current single-center retrospective observational case series was conducted at Shimane University Hospital, Izumo, Japan, as a part of the study protocol "Establishment of New Gonioscopic Methods and Development of Automatic Analysis Algorithms Using 360-degree Gonioscopic Images." The institutional review board (IRB) of Shimane University Hospital reviewed and approved the research (No. 20181124-1). All research adhered to the tenets of the Declaration of Helsinki. The IRB approval did not require each patient to provide written informed consent for publication; instead, the study protocol was posted at the study institutions to notify participants about the study, and they were given the opportunity to opt out from the research.

\section{Participants}

One hundred eight eyes of 75 consecutive subjects with open-angle glaucoma were identified in a search of the medical record data and enrolled; the eyes had 360 degrees of visible TM preoperatively and had undergone $\mu \mathrm{LOT}$ or combined $\mu \mathrm{LOT}$ and cataract surgery. One surgeon (MT) performed all the cataract surgeries and the initial glaucoma surgery, and all had been performed successfully using the gonioscope GS-1 between October 2018 to August 2019 at Shimane University Hospital. Eyes were excluded if they had PAS preoperatively, active uveitis or a history of uveitis, intraocular inflammation, trauma, and an eye surgery that included cataract extraction and laser treatment, which might have affected postoperative PAS formation. All patients were Japanese and data from both eyes were included. In addition to patient age, gender, ocular characteristics of each study eye, and clinical diagnosis, the following examination data were obtained from the medical chart review: visual acuity; findings from slitlamp, manual gonioscopy, and fundus examinations; refractive error by auto-refractometry (RC-5000, Tomey Corporation, Nagoya, Japan); IOP measured by Goldmann applanation tonometry (Haag-Streit, Koniz, Switzerland); visual field mean deviation of the central 30-2 program by Humphrey Field Analyzer (HFA) (Carl Zeiss Meditec, Dublin, CA, USA); axial length and central anterior chamber depth (ACD) measured by optical biometry (OA-2000, Tomey Corporation); and anterior chamber aqueous flare measured by FM-600 laser flare meter (Kowa, Nagoya, Japan). The patients with primary open-angle glaucoma (POAG) had glaucomatous optic nerve damage with a normal-appearing anterior chamber angle and visual function loss on HFA. ${ }^{17}$ The patients with pseudoexfoliation glaucoma had a history of IOP elevation that exceeded $22 \mathrm{mmHg}$ without treatment because of pseudoexfoliation material. ${ }^{18}$ The patients with juvenile openangle glaucoma had increased IOP or a history of IOP elevation exceeding $22 \mathrm{mmHg}$ without treatment, 
glaucomatous optic nerve damage for a primary reason excluding all secondary causes, and visual function loss on perimetry. The patients had been diagnosed between the ages of 3 and 40 years. ${ }^{19}$ The exclusion criteria were the inability to obtain clear GS-1 gonio-photographs, which prevented reliable examination for the presence of PAS. After excluding three eyes of three subjects because of poor-quality images, 105 eyes of 75 participants were analyzed.

\section{Gonioscope GS-I Imaging}

The Gonioscope GS-1 is a recently released anteriorsegment imaging device that covers 360 degrees of the angle and provides true-color gonio-images automatically in a standardized manner in less than 1 minute/eye. Because these images can be analyzed post-hoc, physicians can make detailed observations and magnify any abnormalities. According to our previous reports, ${ }^{20,21}$ after applying topical anesthetic eye drops and using gel coupling, gonio-images of the entire circumference were captured by GS-1 with the participants fixating in primary gaze in a darkened room as during a standard evaluation. The angle was detected using a prism with 16 mirrored facets, which covers about 30 degrees each, and a series of 16 images was acquired as if performing indirect gonioscopy with a Goldmann lens. The instrument then automatically displayed the image with the best focus within the 17 depths of focus for each position. Finally, we acquired the 16 gonio-images with the best focus for each eye. Figure 1A shows a typical merged GS-1 image displayed in a 360-degree view of the angle.

\section{Microhook Ab-interno Trabeculotomy}

A standard surgical procedure was performed with microhooks during $\mu$ LOT. A Swan-Jacob gonioprism lens (Ocular Instruments, Inc., Bellevue, WA) was used to observe the angle opposite to the corneal port, and the microhook was inserted into the anterior chamber through the corneal port. The microhook tip then was inserted into Schlemm's canal and moved circumferentially to incise the inner wall of the canal and TM over 90-degree extents; this also was performed on the opposite side. For postoperative eye drops, $1.5 \%$ levofloxacin (Nipro, Osaka, Japan) and $0.1 \%$ betamethasone (Sanbetason, Santen Pharmaceutical) are applied topically 4 times daily for 3-4 weeks in all cases. Miotic ophthalmic solutions are not used postoperatively. ${ }^{16}$ Regarding the clinical records, all subjects had undergone successful standard $\mu$ LOT procedures, and the incisions were created that extended from 180 to 240 degrees, with at least the following sectors of the TM incised: the superotemporal (ST) to superotemporal-temporal (STT) inferotemporal (IT) to inferoinferior-temporal (IIT) and inferoinferior nasal (IIN) to inferonasal (IN) superonasal-nasal (SNN) to superonasal (SN). Therefore, eight images of the STT, T, ITT, IT, IN, INN, N, and SNN sectors were included in the extend of the $\mu$ LOT incisions (Figure 1B).

\section{Postoperative PAS Evaluations with GS-I Gonio-Images}

We assessed the prevalence of PAS formation after trabeculotomy and the rates of postoperative PAS formation in the total angle circumference both within the extent of the incision and outside of the incision in the 16 GS-1 gonioimages of each eye. An ophthalmologist (MM) who was masked to the study and familiar with the angle determinations using the GS-1 evaluated the PAS formation in each gonio-photograph. We then calculated the rate of PAS formation as follows: total $=\mathrm{X}$ images $/ 16$ images; rate of PAS formation in the extent of the $\mu$ LOT incision $=$ $\mathrm{Y}$ images/8 images; and the rate of PAS formation outside of the $\mu \mathrm{LOT}$ incision $=\mathrm{Z}$ images $/ 8$ images. Figure $1 \mathrm{~A}$ shows a representative image of the GS-1 with PAS formation postoperatively, and it was determined that $X=5$, $\mathrm{Y}=5$, and $\mathrm{Z}=0$ in this case.

\section{Statistical Analysis}

All statistical analyses were performed using the JMP Pro version 14.2 statistical software (SAS Institute, Inc., Cary, NC, USA). The clinical and demographic characteristics were expressed as the means and standard deviations (SD) for continuous variables or by the number and frequency of discrete variables. The differences in the continuous variables were evaluated statistically between preoperatively and postoperatively in the study population using the paired $t$-test. The incidence of PAS formation and rates after $\mu \mathrm{LOT}$ were classified based on the categorical variables in the demographic data and then analyzed using the Pearson's chi-square test for categorical data and the Student's $t$-test for continuous data or the Pearson's chisquare test for categorical data and one-way analysis of variance for continuous data. The paired $t$-test also was used to analyze the differences between the PAS formation rates within and outside of the incisions. Moreover, the possible associations among the PAS rate and various 
A

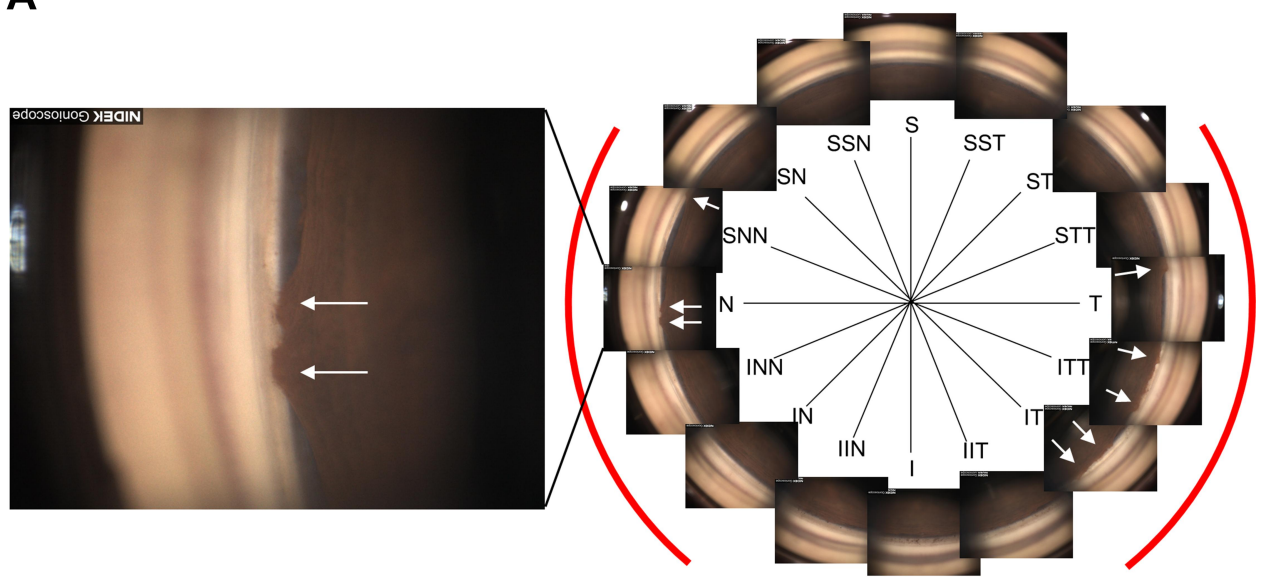

B

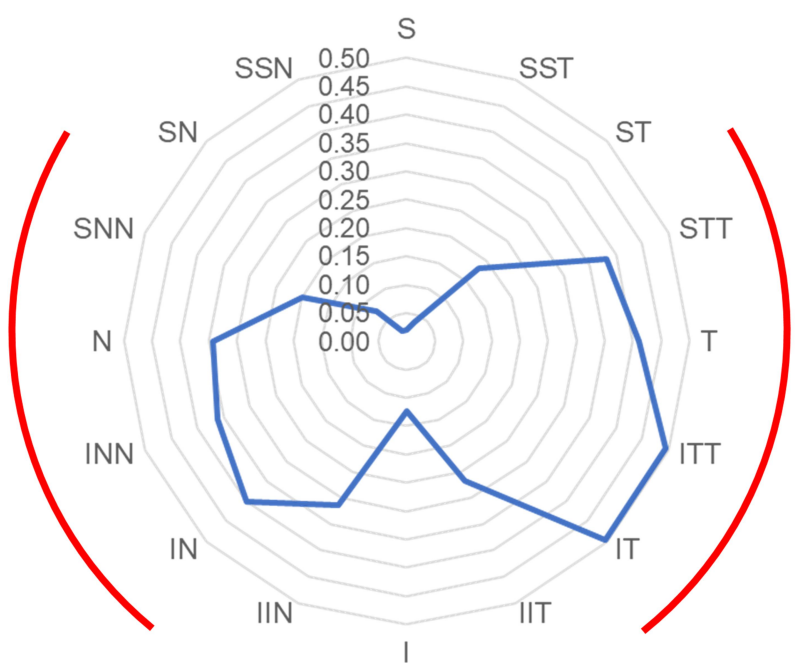

Figure I Representative gonioscope GS-I images with PAS formation after $\mu$ LOT in a 360-degree view of the angle, and the distribution of the average PAS formation rate in each angle position.

Notes: (A) The typical merged GS-I image after $\mu$ LOT displayed in a 360-degree view of the angle is shown on the right side, in which the ocular features are seen as in direct gonioscopy, with the extent of the incision indicated in red. Each PAS formation is indicated by a white arrow. The image shows the left eye of a patient with primary open-angle glaucoma, for whom five gonio-images show the extent of the PAS within the incision. In addition, a GS-I image of the extent of the incision, in which PAS is present, is shown on the left. (B) The radar chart shows the distribution of the average PAS formation rates in each sector. PAS appears to form frequently in the incision. Abbreviations: PAS, peripheral anterior synechia; $\mu$ LOT, microhook ab interno trabeculotomy; S, superior; SST, superior-superior-temporal; ST, superior-temporal; STT, superior-temporal-temporal; T, temporal; ITT, inferior-temporal-temporal; IT, inferior-temporal; IIT, inferior-inferior-temporal; I, inferior; IIN, inferior-inferior-nasal; IN, inferior-nasal; INN, inferior-nasal-nasal; N, nasal; SNN, superior-nasal-nasal; SN, superior-nasal; SSN, superior-superior-nasal.

variables were analyzed by a simple linear regression model and a mixed-effect model. $\mathrm{P}<0.05$ was considered statistically significant.

\section{Results}

A total of 105 eyes of 75 participants were analyzed. Of the 105 eyes with an open angle, 68 had POAG, 27 had pseudoexfoliation glaucoma, and 10 had juvenile openangle glaucoma. Seventy-nine eyes had undergone $\mu$ LOT combined with uncomplicated phacoemulsification and intraocular lens implantation, and the other 26 eyes had undergone $\mu$ LOT alone. Table 1 shows the continuous variables preoperatively and postoperatively in the study subjects. All participants were Japanese. The mean age $( \pm \mathrm{SD})$ was $64.1 \pm 15.6$ years. PAS formed in $90(86 \%)$ eyes postoperatively. The mean numbers $( \pm \mathrm{SD})$ of images that showed postoperative PAS formation in all eyes were $4.1 \pm 3.2$, within the $\mu \mathrm{LOT}$ incision $3.1 \pm 2.3$, and outside of the $\mu \mathrm{LOT}$ incision $1.0 \pm 1.3$. Figure 1B shows the distribution of the average PAS formation rate in each sector of the GS-1 gonio-images. 
Table I Continuous Preoperative and Postoperative Variables

\begin{tabular}{|c|c|c|c|}
\hline Variable & $\begin{array}{l}\text { Preoperative } \\
\text { Conditions }(n=105)\end{array}$ & $\begin{array}{l}\text { Postoperative } \\
\text { Conditions }(n=105)\end{array}$ & $\mathbf{p}^{*}$ \\
\hline \multicolumn{4}{|l|}{ Baseline characteristics } \\
\hline Age, years, mean \pm SD (range) & $64.1 \pm 15.6(11-87)$ & NA & \\
\hline Mean Shaffer grade, mean \pm SD (range) & $3.5 \pm 0.72(I-4)$ & NA & \\
\hline Axial length, mm, mean $\pm \mathrm{SD}$ (range) & $24.9 \pm 2.0(21.2-30.7)$ & NA & \\
\hline Visual field mean deviation, $\mathrm{dB}$, mean $\pm \mathrm{SD}$ (range) & $-12.6 \pm 8.4(-29.7|-| .16)$ & NA & \\
\hline Postoperative days, mean \pm SD (range) & 0 & $225 \pm 226(14-1021)$ & $<0.01$ \\
\hline LogMAR best corrected visual acuity, mean \pm SD (range) & $0.19 \pm 0.43(-0.08-2.30)$ & $0.11 \pm 0.39(-0.08-2.30)$ & $<0.01$ \\
\hline Intraocular pressure, $\mathrm{mmHg}$, mean $\pm \mathrm{SD}$ (range) & $20.7 \pm 6.7(|2.0-5| .0)$ & $13.7 \pm 3.7(6-2 \mid)$ & $<0.01$ \\
\hline Central anterior chamber depth, $\mathrm{mm}$, mean $\pm \mathrm{SD}$ (range) & $3.28 \pm 0.42(2.06-4.77)$ & $3.69 \pm 0.67(2.33-5.4 I)$ & $<0.01$ \\
\hline Aqueous flare, $\mathrm{pc} / \mathrm{ms}$, mean $\pm \mathrm{SD}$ (range) & $9.1 \pm 5.0(2.6-27.9)$ & $15.3 \pm 14.5(2.3-121.3)$ & $<0.01$ \\
\hline Topical glaucoma medications, mean \pm SD (range) & $3.3 \pm 0.9(I-5)$ & $2.7 \pm 0.8(0-4)$ & $<0.01$ \\
\hline \multicolumn{4}{|l|}{ Primary outcome variables } \\
\hline Peripheral anterior synechiae formation, number (\%) & 0 & $90(86)$ & $<0.01$ \\
\hline $\begin{array}{l}\text { Images of peripheral anterior synechiae formation in total, mean } \pm \text { SD } \\
\text { (range) }\end{array}$ & 0 & $4.1 \pm 3.2(0-14)$ & $<0.01$ \\
\hline $\begin{array}{l}\text { Images of peripheral anterior synechiae formation in incision extent of } \\
\mu \mathrm{LOT} \text {, mean } \pm \mathrm{SD} \text { (range) }\end{array}$ & 0 & $3.1 \pm 2.3(0-8)$ & $<0.01$ \\
\hline $\begin{array}{l}\text { Images of peripheral anterior synechiae formation in non-incision extent } \\
\text { of } \mu \text { LOT, mean } \pm S D \text { (range) }\end{array}$ & 0 & I.0 $.1 .3(0-7)$ & $<0.01$ \\
\hline
\end{tabular}

Note: Statistical significance was analyzed by the paired $t$-test $(*)$.

Abbreviations: SD, standard deviation; $\mu \mathrm{LOT}$, microhook ab interno trabeculotomy; n, number of eyes; NA, not applicable; dB, decibels; logMAR, logarithm of the minimum angle of resolution; $\mathrm{pc} / \mathrm{ms}$, photon counts per millisecond.

Table 2 shows the postoperative PAS formation frequencies and rates classified according to the categorical variables in the demographic data. In the group that underwent the combined surgery, the total PAS rate was significantly higher than that in the $\mu$ LOT alone group $(\mathrm{p}=0.03)$. The PAS frequencies also differed significantly among the glaucoma types $(\mathrm{p}=0.03)$. Thus, we assumed that regardless of whether the combined surgery or solo surgery was performed, the glaucoma type could be related to PAS formation.

Table 3 shows the possible associations among the PAS rates and various continuous variables analyzed by the simple linear regression model. The preoperative mean Shaffer grade, preoperative axial length, and preoperative and postoperative central ACD were negatively associated with the total PAS rate ( $<<0.05$, respectively). The preoperative central ACD had a higher negative regression coefficient with the total PAS rate, with a lower $p$ value than the postoperative central ACD. The postoperative days and preoperative topical glaucoma medications were positively correlated with the total PAS rate $(\mathrm{p}<0.05$ for both comparisons). Thus, we considered that the preoperative mean Shaffer grade, preoperative axial length, preoperative central ACD, postoperative days, and preoperative topical glaucoma medications also could be related to PAS formation.

Table 4 shows the possible associations among the PAS rate and various variables analyzed by the mixed-effect model that was performed with the eye as the unit of analysis, with a fixed effect for the possible related factors detected in Tables 2 and 3, and with crossed random effects to adjust for a correlation with the fellow eyes. We also added the preoperative IOP to the analysis as a fixed effect, which should be strongly correlated with the preoperative topical glaucoma medications. Finally, we found that the total PAS rate and the rate within the incision were correlated positively with the postoperative days ( $\mathrm{p}=0.01$ and 0.004 , respectively), that the extent outside of the incision was correlated positively with $\mu \mathrm{LOT}$ alone, and that there was a negative correlation with the preoperative central ACD $(\mathrm{p}=0.048$ and 0.04 , respectively).

\section{Discussion}

The current study investigated the prevalence, location, and related characteristics of PAS formation after $\mu$ LOT using gonioscope GS-1 images that should the details of the entire circumference of the angle and analyzed their 
Table 2 PAS Formation Frequencies and Rates After $\mu$ lot Classified by Categorical Variables in Demographic Data

\begin{tabular}{|c|c|c|c|c|c|}
\hline Variable & $\begin{array}{c}\text { PAS } \\
\text { Number } \\
(\%)\end{array}$ & $\begin{array}{c}\text { Total PAS Rate, } \\
\text { Mean } \pm \text { SD }\end{array}$ & $\begin{array}{l}\text { Rate Within } \mu \text { LOT } \\
\text { Incision, Mean } \pm \text { SD }\end{array}$ & $\begin{array}{l}\text { Rate Outside of } \mu \text { LOT } \\
\text { Incision, Mean } \pm \text { SD }\end{array}$ & $\mathbf{p}^{\ddagger}$ \\
\hline $\begin{array}{l}\text { All study subjects } \\
(\mathrm{n}=105)\end{array}$ & $90(86)$ & $0.26 \pm 0.20$ & $0.39 \pm 0.29$ & $0.13 \pm 0.16$ & $<0.0001$ \\
\hline $\begin{array}{l}\text { Sex } \\
\text { Male }(n=60) \\
\text { Female }(n=45) \\
p^{*}\end{array}$ & $\begin{array}{c}53(88) \\
37(82) \\
0.38\end{array}$ & $\begin{array}{c}0.26 \pm 0.18 \\
0.26 \pm 0.23 \\
0.96\end{array}$ & $\begin{array}{c}0.39 \pm 0.27 \\
0.38 \pm 0.32 \\
0.81\end{array}$ & $\begin{array}{c}0.13 \pm 0.15 \\
0.14 \pm 0.18 \\
0.74\end{array}$ & $\begin{array}{l}<0.0001 \\
<0.0001\end{array}$ \\
\hline $\begin{array}{l}\text { Right or left eye } \\
\text { Right eye }(n=53) \\
\text { Left eye }(n=52) \\
P^{*}\end{array}$ & $\begin{array}{c}46(87) \\
44(85) \\
0.75\end{array}$ & $\begin{array}{c}0.26 \pm 0.20 \\
0.26 \pm 0.20 \\
0.96\end{array}$ & $\begin{array}{c}0.38 \pm 0.28 \\
0.39 \pm 0.30 \\
0.84\end{array}$ & $\begin{array}{c}0.14 \pm 0.17 \\
0.13 \pm 0.16 \\
0.76\end{array}$ & $\begin{array}{l}<0.0001 \\
<0.0001\end{array}$ \\
\hline $\begin{array}{l}\mu \text { LOT combined with } \\
\text { cataract surgery } \\
\text { Yes }(n=79) \\
\text { No }(n=26) \\
p^{*}\end{array}$ & $\begin{array}{c}65(82) \\
25(96) \\
0.08\end{array}$ & $\begin{array}{c}0.23 \pm 0.20 \\
0.33 \pm 0.19 \\
0.03\end{array}$ & $\begin{array}{c}0.36 \pm 0.30 \\
0.48 \pm 0.24 \\
0.07\end{array}$ & $\begin{array}{c}0.11 \pm 0.15 \\
0.19 \pm 0.19 \\
0.09\end{array}$ & $\begin{array}{l}<0.0001 \\
<0.0001\end{array}$ \\
\hline $\begin{array}{l}\text { Glaucoma type } \\
\text { POAG }(n=68) \\
\text { PEG }(n=27) \\
\text { JOAG }(n=10) \\
P^{\dagger}\end{array}$ & $\begin{array}{c}62(91) \\
19(70) \\
9(90) \\
0.03\end{array}$ & $\begin{array}{c}0.25 \pm 0.17 \\
0.26 \pm 0.24 \\
0.29 \pm 0.25 \\
0.86\end{array}$ & $\begin{array}{c}0.39 \pm 0.27 \\
0.38 \pm 0.35 \\
0.38 \pm 0.28 \\
0.96\end{array}$ & $\begin{array}{c}0.11 \pm 0.12 \\
0.15 \pm 0.20 \\
0.20 \pm 0.26 \\
0.23\end{array}$ & $\begin{array}{c}<0.0001 \\
0.0005 \\
0.02\end{array}$ \\
\hline
\end{tabular}

Notes: Statistical significance was analyzed by the chi-square test for categorical data and the Student's $t$-test for continuous data $(*)$; by the chi-square test for categorical data and one-way analysis of variance for continuous data $\left({ }^{\dagger}\right)$; by the paired $t$-test $\left(^{\ddagger}\right)$.

Abbreviations: PAS, peripheral anterior synechiae; $\mu$ LOT, microhook ab-interno trabeculotomy; $n$, number of eyes; POAG, primary open angle glaucoma; PEG, pseudoexfoliation glaucoma; JOAG, juvenile open angle glaucoma; SD, standard deviation.

effects on clinical variables including the IOP and number of antiglaucoma medications.

Compared to baseline, examinations 225 days postoperatively showed that the IOP decreased significantly by $34 \%$ from 20.7 to $13.7 \mathrm{mmHg}$. The number of antiglaucoma medications also decreased significantly by $18 \%$ from 3.3 to 2.7 . The 6-month postoperative examination showed that the IOP decreased significantly by $43 \%$ from 25.9 to $14.7 \mathrm{mmHg}$ as the result of $\mu \mathrm{LOT}$ alone and the number of antiglaucoma medications decreased by $15 \%$ from 3.3 to $2.8 .{ }^{14}$ At the 9.5 -month examination after $\mu$ LOT combined with cataract surgery, the IOP decreased significantly by $28 \%$ from 16.4 to $11.8 \mathrm{mmHg}$, and the number of antiglaucoma medications decreased by $13 \%$ from 2.4 to $2.1{ }^{15}$ Moreover, a recent study reported the significant continuous effects of the combined procedures on reducing the IOP and the number of antiglaucoma medications at all time points between 1 and 6 months postoperatively. ${ }^{10}$ Therefore, similar to previous reports, $\mu \mathrm{LOT}$ alone or $\mu \mathrm{LOT}$ combined with cataract surgery resulted in significant decreases in the IOP and number of antiglaucoma medications during follow-up examinations.

We initially evaluated PAS formation after trabeculotomy in detail using GS-1 gonio-images that cover 360 degrees of the angle. Tables 1 and 2 show that PAS developed after $\mu \mathrm{LOT}$ in $86 \%$ of patients and the total formation rate was 0.26 . In addition, the rate was significantly higher within the $\mu \mathrm{LOT}$ incision (rate, 0.39 ) than outside of the incision (rate, 0.13). Figure 1B is a radar chart that shows the distribution of the average PAS formation rates in each sector on the 360-degree gonioimages of the GS-1. The results could be generalized in PAS formation after the other trabeculotomy-related glaucoma surgeries. Moreover, the methodology would be useful to objectively assess the postoperative angle changes.

The results of the current analyses indicated that the total PAS rate and the rate within the incision may be associated with the postoperative days, that PAS formation 
Table 3 Possible Associations Among PAS Rates and Various Continuous Variables Analyzed by the Simple Linear Regression Model

\begin{tabular}{|c|c|c|c|c|c|c|c|c|c|c|c|c|}
\hline \multirow[t]{3}{*}{ Variable } & \multicolumn{6}{|c|}{ Preoperative Conditions $(n=105)$} & \multicolumn{6}{|c|}{ Postoperative Conditions $(n=105)$} \\
\hline & \multicolumn{2}{|c|}{$\begin{array}{l}\text { Total PAS } \\
\text { Rate }\end{array}$} & \multicolumn{2}{|c|}{$\begin{array}{c}\text { PAS Rate } \\
\text { Within } \mu \text { LOT } \\
\text { Incision }\end{array}$} & \multicolumn{2}{|c|}{$\begin{array}{c}\text { PAS Rate } \\
\text { outside of } \\
\mu \text { LOT Incision }\end{array}$} & \multicolumn{2}{|c|}{ Total PAS rate } & \multicolumn{2}{|c|}{$\begin{array}{c}\text { PAS Rate } \\
\text { Within } \mu \text { LOT } \\
\text { Incision }\end{array}$} & \multicolumn{2}{|c|}{$\begin{array}{c}\text { PAS Rate } \\
\text { Outside of } \\
\text { MLOT Incision }\end{array}$} \\
\hline & $\begin{array}{l}r(95 \% \\
\text { CI) }\end{array}$ & $\mathbf{p}^{*}$ & $\begin{array}{l}r(95 \% \\
\text { CI) }\end{array}$ & $\mathbf{p}^{*}$ & $\begin{array}{c}r(95 \% \\
\mathrm{CI})\end{array}$ & $\mathbf{p}^{*}$ & $\begin{array}{c}r(95 \% \\
\mathrm{Cl})\end{array}$ & $\mathbf{p}^{*}$ & $\begin{array}{c}r(95 \% \\
C I)\end{array}$ & $\mathbf{p}^{*}$ & $\begin{array}{l}r(95 \% \\
\text { CI) }\end{array}$ & $\mathbf{p}^{*}$ \\
\hline Age (/year) & -0.0016 & 0.20 & -0.0016 & 0.38 & -0.0016 & 0.12 & NA & NA & NA & NA & NA & NA \\
\hline Mean Shaffer grade & -0.058 & 0.03 & -0.070 & 0.08 & -0.046 & 0.04 & NA & NA & NA & NA & NA & NA \\
\hline Axial length $(/ \mathrm{mm})$ & -0.023 & 0.02 & -0.028 & 0.053 & -0.019 & 0.02 & NA & NA & NA & NA & NA & NA \\
\hline $\begin{array}{l}\text { Visual field mean } \\
\text { deviation }(/ d B)\end{array}$ & -0.0012 & 0.62 & -0.0021 & 0.55 & -0.00028 & 0.89 & NA & NA & NA & NA & NA & NA \\
\hline $\begin{array}{l}\text { Postoperative days } \\
\text { (/day) }\end{array}$ & NA & NA & NA & NA & NA & NA & 0.00022 & 0.01 & 0.00034 & $<0.01$ & 0.000096 & 0.18 \\
\hline $\begin{array}{l}\text { LogMAR best-corrected } \\
\text { visual acuity }\end{array}$ & 0.060 & 0.18 & 0.12 & 0.07 & 0.00048 & 0.99 & 0.036 & 0.48 & 0.093 & 0.21 & -0.020 & 0.63 \\
\hline $\begin{array}{l}\text { Intraocular pressure } \\
(/ \mathrm{mmHg})\end{array}$ & 0.00046 & 0.88 & 0.0050 & 0.25 & -0.0040 & 0.10 & -0.0055 & 0.23 & -0.0052 & 0.43 & -0.0056 & 0.13 \\
\hline $\begin{array}{l}\text { Central anterior } \\
\text { chamber depth }(/ \mathrm{mm})\end{array}$ & -0.17 & $<0.01$ & -0.19 & $<0.01$ & -0.15 & $<0.01$ & -0.068 & 0.02 & -0.096 & 0.03 & -0.040 & 0.10 \\
\hline Aqueous flare (/pc/ms) & 0.0012 & 0.77 & 0.00071 & 0.90 & 0.0016 & 0.63 & -0.00050 & 0.71 & -0.0018 & 0.38 & 0.00075 & 0.50 \\
\hline $\begin{array}{l}\text { Topical glaucoma } \\
\text { medications }\end{array}$ & 0.062 & $<0.01$ & 0.097 & $<0.01$ & 0.027 & 0.12 & 0.024 & 0.32 & 0.056 & 0.11 & -0.0066 & 0.74 \\
\hline
\end{tabular}

Note: Possible association was analyzed by the simple linear regression model $(*)$.

Abbreviations: PAS, peripheral anterior synechiae; $n$, number of eyes; $\mu$ LOT, microhook ab-interno trabeculotomy; SD, standard deviation; $r$, regression coefficient; NA, not applicable; logMAR, logarithm of the minimum angle of resolution; $\mathrm{dB}$, decibels; $\mathrm{Cl}$, confidence interval; pc/ms, photon counts per millisecond.

outside of the incision may be associated with the combined surgery or not and with the preoperative central ACD, which might help elucidate the etiology and pathophysiology of PAS formation in different conditions. Synechiae are thought to form during two different conditions, ie, in association with inflammation and cellular proliferation and in association with non-proliferation. The pathophysiology of the former would be related to inflammatory cells, fibrin, and protein deposition, which stimulate the formation of adhesions between structures. However, in the latter condition, PAS may result from iris apposition on the TM, which includes trauma and increased IOP. ${ }^{22}$ Therefore, the total PAS and PAS within the $\mu$ LOT incision would develop gradually over time postoperatively because of the continuous increased outflow into Schlemm's canal within the incision, which induces iris apposition on the TM. Inflammation associated with surgery might contribute to PAS formation; however, we did not detect this because aqueous flare, which is used to estimate postoperative inflammation, was not associated significantly with the PAS rate. Accordingly, inflammation can be associated with formation of PAS during early postoperative periods rather than the gradual increase of PAS during later periods. While aqueous outflow into the area of the TM that was not incised during $\mu$ LOT should not increase, PAS in the nonincised area may have developed due to the shallow postoperative ACD, which also stimulates iris apposition on the TM. Therefore, a deep preoperative ACD and combined cataract surgery, which deepen the postoperative ACD, would be significantly negatively correlated with the PAS rate in the current study. Accordingly, PAS within the incision and PAS in the non-incised area might result from different causes.

We did not detect a correlation between the rate of PAS formation and a therapeutic effect by $\mu \mathrm{LOT}$; however, 
Table 4 Possible Associations Among PAS Rate and Various Variables Analyzed by the Mixed-Effect Model

\begin{tabular}{|c|c|c|c|c|c|c|}
\hline \multirow[t]{2}{*}{ Variable } & \multicolumn{2}{|c|}{ Total PAS Rate $(n=105)$} & \multicolumn{2}{|c|}{$\begin{array}{l}\text { PAS Rate Within } \mu \text { LOT } \\
\text { Incision }(n=105)\end{array}$} & \multicolumn{2}{|c|}{$\begin{array}{l}\text { PAS Rate Outside of } \\
\mu \text { LOT Incision }(n=105)\end{array}$} \\
\hline & $\begin{array}{c}\text { Estimate }(95 \% \\
\mathrm{Cl})\end{array}$ & $\mathbf{p}^{*}$ & $\begin{array}{c}\text { Estimate }(95 \% \\
\text { Cl) }\end{array}$ & $\mathbf{p}^{*}$ & $\begin{array}{c}\text { Estimate }(95 \% \\
\text { Cl) }\end{array}$ & $\mathbf{p}^{*}$ \\
\hline Postoperative days (/day) & 0.00022 & 0.01 & 0.00037 & 0.004 & 0.000078 & 0.29 \\
\hline $\mu \mathrm{LOT}$ combined with cataract surgery (/yes) & 0.062 & 0.06 & 0.064 & 0.17 & 0.054 & 0.048 \\
\hline $\begin{array}{l}\text { Preoperative central anterior chamber depth } \\
(/ \mathrm{mm})\end{array}$ & -0.11 & 0.07 & -0.12 & 0.19 & -0.11 & 0.04 \\
\hline Preoperative topical glaucoma medications & 0.031 & 0.19 & 0.060 & 0.08 & 0.0056 & 0.77 \\
\hline Axial length $(/ \mathrm{mm})$ & -0.013 & 0.28 & -0.014 & 0.41 & -0.0093 & 0.35 \\
\hline Mean Shaffer grade & -0.033 & 0.35 & -0.054 & 0.29 & -0.0079 & 0.79 \\
\hline Preoperative intraocular pressure $(/ \mathrm{mmHg})$ & 0.0028 & 0.42 & 0.0092 & 0.07 & -0.0035 & 0.23 \\
\hline Glaucoma type (JOAG/POAG) & 0.016 & 0.78 & 0.012 & 0.89 & 0.022 & 0.65 \\
\hline Glaucoma type (PEG/POAG) & -0.0052 & 0.90 & -0.024 & 0.70 & 0.0086 & 0.81 \\
\hline
\end{tabular}

Notes: Possible association was analyzed by the mixed-effect model with crossed random effects to adjust for correlation between fellow eyes $(*)$.

Abbreviations: PAS, peripheral anterior synechiae; $\mu$ LOT, microhook ab-interno trabeculotomy; POAG, primary open angle glaucoma; JOAG, juvenile open angle glaucoma; PEG, pseudoexfoliation glaucoma; $\mathrm{n}$, number of eyes; $\mathrm{Cl}$, confidence interval.

several studies have reported a relationship between PAS and increased IOP or attenuation of the IOP-lowering effect as a result of treatment. ${ }^{23-25}$ One reason may be that the total PAS rate (rate, 0.26) was not sufficient to increase the IOP. Moreover, the actual extent of the PAS would be shorter than the estimated PAS rate because we calculated the rate based on the presence of PAS in each gonio-image, which could be overestimated. Thus, we should study this hypothesis in a future study that measures the actual extent of the PAS.

The current study had other limitations. First, postoperative observation was performed at only one point for a relatively a short period and the time points of observation were not consistent because of the retrospective observational nature of the study. Although we did not detect a significant difference in the relationship, we cannot deny that possibility. Second, the study included relatively few patients, so the statistical power might have been insufficient to detect all factors involved in PAS formation. Finally, our study might have limited generalizability because of the exclusion of the poor-quality images. Therefore, further studies are needed to reach a definitive conclusion about the relationships between PAS after $\mu$ LOT and the IOP-lowering effect.

\section{Conclusion}

The current study identified postoperative PAS formation after $\mu \mathrm{LOT}$ in most patients and the rate was significantly higher within the incision compared with the rate outside of the incision. A radar chart also showed the distribution of the average PAS formation rate in each sector. Moreover, we showed that the total PAS rate and the rate within the incision may be associated with the postoperative days, that in the area not incised there was a possible association with combined surgery or not and with preoperative central ACD in the mixed-effect model analysis, which suggested that PAS within the incision and the PAS in the area outside the incision would develop from different causes. However, while further studies are needed, the current study contributes knowledge about the surgical outcomes of trabeculotomy-related glaucoma surgeries.

\section{Author Contributions}

All authors made a significant contribution to the work reported, whether that is in the conception, study design, execution, acquisition of data, analysis and interpretation, or in all these areas; took part in drafting, revising or critically reviewing the article; gave final approval of the version to be published; have agreed on the journal to 
which the article has been submitted; and agree to be accountable for all aspects of the work.

\section{Funding}

This work was supported by the Japan Society for the Promotion of Science (JSPS) KAKENHI Grant Number 20K18382 (MM).

\section{Disclosure}

Dr Masato Matsuo reports grants from the Japan Society for the Promotion of Science (JSPS) KAKENHI, personal fees from Inami \& Co., Ltd., during the conduct of the study. Ms Yuina Inomata reports grants from the Japan Society for the Promotion of Science, personal fees from Inami \& Co., Ltd., during the conduct of the study. Mrs Nana Kozuki reports grants from the Japan Society for the Promotion of Science (JSPS) KAKENHI, personal fees from Inami \& Co., Ltd, during the conduct of the study. Prof. Dr Masaki Tanito reports personal fees from Inami, during the conduct of the study; personal fees from Inami, outside the submitted work.

\section{References}

1. Flaxman SR, Bourne RRA, Resnikoff S, et al. Global causes of blindness and distance vision impairment 1990-2020: a systematic review and meta-analysis. Lancet Glob Health. 2017;5(12):e1221e34. doi:10.1016/s2214-109x(17)30393-5

2. Tham YC, Li X, Wong TY, et al. Global prevalence of glaucoma and projections of glaucoma burden through 2040: a systematic review and meta-analysis. Ophthalmology. 2014;121(11):2081-2090. doi:10.1016/ j.ophtha.2014.05.013

3. Ernest PJ, Schouten JS, Beckers HJ, et al. An evidence-based review of prognostic factors for glaucomatous visual field progression. Ophthalmology. 2013;120(3):512-519. doi:10.1016/j. ophtha.2012.09.005

4. Lavia C, Dallorto L, Maule M, et al. Minimally-invasive glaucoma surgeries (MIGS) for open angle glaucoma: a systematic review and meta-analysis. PLoS One. 2017;12(8):e0183142. doi:10.1371/journal. pone. 0183142

5. Kaplowitz K, Bussel II, Honkanen R, et al. Review and meta-analysis of ab-interno trabeculectomy outcomes. Br J Ophthalmol. 2016;100 (5):594-600. doi:10.1136/bjophthalmol-2015-307131

6. Sieck EG, Epstein RS, Kennedy JB, et al. Outcomes of Kahook dual blade goniotomy with and without phacoemulsification cataract extraction. Ophthalmol Glaucoma. 2018;1(1):75-81. doi:10.1016/j. ogla.2018.06.006

7. Grover DS, Godfrey DG, Smith O, et al. Gonioscopy-assisted transluminal trabeculotomy, ab interno trabeculotomy: technique report and preliminary results. Ophthalmology. 2014;121(4):855-861. doi:10.1016/j.ophtha.2013.11.001

8. Wang SY, Singh K, Stein JD, et al. Ocular antihypertensive medication use after iStent implantation concurrent with cataract surgery vs cataract surgery alone in a large US Health Care Claims Database. JAMA Ophthalmol. 2019;137(1):21-27. doi:10.1001/ jamaophthalmol.2018.4461
9. Tanito M, Matsuo M. Ab-interno trabeculotomy-related glaucoma surgeries. Taiwan J Ophthalmol. 2019;9(2):67-71. doi:10.4103/tjo. tjo_38_19

10. Omoto T, Fujishiro T, Asano-Shimizu K, et al. Comparison of the short-term effectiveness and safety profile of ab interno combined trabeculotomy using 2 types of trabecular hooks. Jpn J Ophthalmol. 2020;64(4):407-413. doi:10.1007/s10384-020-00750-3

11. Hepsen IF, Guler E, Kumova D, et al. Efficacy of modified 360-degree Suture trabeculotomy for pseudoexfoliation glaucoma. J Glaucoma. 2016;25(1):e29-34. doi:10.1097/ijg.0000000000000214

12. Hepsen IF, Guler E, Yalcin NG, et al. Modified 360-degree suture trabeculotomy for pseudoexfoliation glaucoma: 12-month results. J Glaucoma. 2016;25(4):e408-12. doi:10.1097/ ijg.00000000000000358

13. Tanito M. Microhook ab interno trabeculotomy, a novel minimally invasive glaucoma surgery. Clin Ophthalmol. 2018;12:43-48. doi:10.2147/opth.s152406

14. Tanito M, Sano I, Ikeda Y, et al. Short-term results of microhook ab interno trabeculotomy, a novel minimally invasive glaucoma surgery in Japanese eyes: initial case series. Acta Ophthalmol. 2017;95(5): e354-e60. doi:10.1111/aos.13288

15. Tanito M, Ikeda Y, Fujihara E. Effectiveness and safety of combined cataract surgery and microhook ab interno trabeculotomy in Japanese eyes with glaucoma: report of an initial case series. Jpn J Ophthalmol. 2017;61(6):457-464. doi:10.1007/s10384-017-0531-z

16. Khawaja A, American Academy of Ophthalmology. EyeWiki. Primary open-angle glaucoma;2020. Available from: https://eyewiki. aao.org/Primary_Open-Angle_Glaucoma. Accessed October 29, 2020.

17. Topouzis F, Founti P, Yu F, et al. Twelve-year incidence and baseline risk factors for pseudoexfoliation: the Thessaloniki Eye Study (An American Ophthalmological Society Thesis). Am J Ophthalmol. 2019;206:192-214. doi:10.1016/j.ajo.2019.05.005

18. Feng S, American Academy of Ophthalmology. EyeWiki. Juvenile open angle glaucoma;2020. Available from: https://eyewiki.aao.org/ Juvenile_open_angle_glaucoma. Accessed October 29, 2020.

19. De Giusti A, Pajaro S, Tanito M Automatic pigmentation grading of the trabecular meshwork in gonioscopic images. Proc of Computational Pathology and Ophthalmic Medical Image Analysis (COMPAY-OMIA 2018). Springer International Publishing; 2018: 193-200

20. Matsuo M, Pajaro S, De Giusti A, et al. Automated anterior chamber angle pigmentation analyses using $360^{\circ}$ gonioscopy. $\mathrm{Br}$ $J$ Ophthalmol. 2019;104(5):636-641. doi:10.1136/bjophthalmol2019-314320

21. Pittner A. American Academy of Ophthalmology. EyeWiki. Synechiae; 2020. Available from: https://eyewiki.aao.org/Synechiae. Accessed October 29, 2020.

22. Tanito M, Manabe K, Mochiji M, et al. Comparison of anterior chamber flare among different glaucoma surgeries. Clin Ophthalmol. 2019;13:1609-1612. doi:10.2147/opth.s219715

23. Rouhiainen HJ, Terasvirta ME, Tuovinen EJ. Peripheral anterior synechiae formation after trabeculoplasty. Arch Ophthalmol. 1988;106(2):189-191. doi:10.1001/archopht.1988.01060130199025

24. Mahajan D, Sharma R, Garg SP, et al. Clinical profile of uveitis-related ocular hypertension. Int Ophthalmol. 2014;34 (6):1221-1226. doi:10.1007/s10792-014-0008-8

25. Daniel E, Pistilli M, Kothari S, et al. Risk of ocular hypertension in adults with noninfectious uveitis. Ophthalmology. 2017;124 (8):1196-1208. doi:10.1016/j.ophtha.2017.03.041 


\section{Publish your work in this journal}

Clinical Ophthalmology is an international, peer-reviewed journal covering all subspecialties within ophthalmology. Key topics include: Optometry; Visual science; Pharmacology and drug therapy in eye diseases; Basic Sciences; Primary and Secondary eye care; Patient Safety and Quality of Care Improvements. This journal is indexed on PubMed

Submit your manuscript here: https://www.dovepress.com/clinical-ophthalmology-journal
Central and CAS, and is the official journal of The Society of Clinical Ophthalmology (SCO). The manuscript management system is completely online and includes a very quick and fair peer-review system, which is all easy to use. Visit http://www.dovepress.com/ testimonials.php to read real quotes from published authors. 